\title{
Numerical Solution of Volterra Integral Equations with Weakly Singular Kernel Based on the DE-Sinc Method*
}

\author{
Masatake Mori, Ahniyaz Nurmuhammad ${ }^{\dagger, \ddagger}$ and Takefumi Murai \\ Department of Mathematical Sciences \\ Tokyo Denki University \\ Hatoyama-cho, Hiki-gun, Saitama 350-0394, Japan \\ ${ }^{\ddagger}$ E-mail: nur@r.dendai.ac.jp
}

Received April 12, 2007

Revised September 21, 2007

\begin{abstract}
A method for numerical solution of Volterra integral equations of the second kind with a weakly singular kernel based on the double exponential (DE) transformation is proposed. In this method we first express the approximate solution in the form of a Sinc expansion based on the double exponential transformation by Takahasi and Mori in 1974 followed by collocation at the Sinc points. We also apply the DE formula to the kernel integration. In every sample equation a numerical solution with very high accuracy is obtained and a nearly exponential convergence rate $\exp (-c M / \log M), c>0$ in the error is observed where $M$ is a parameter representing the number of terms in the Sinc expansion. We compared the result with the one based on the single exponential (SE) transformation by Riley in 1992 which made us confirm the high efficiency of the present method.
\end{abstract}

Key words: double exponential transformation, DE transformation, integral equation, Sinc method, weakly singular kernel

\section{Introduction}

In the present paper we consider a Volterra integral equation of the second kind with a weakly singular kernel of the form

$$
y(x)=g(x)+L^{\alpha} y(x), \quad x \geq 0,
$$

where $L^{\alpha}$ denotes a weakly singular linear Volterra operator

$$
L^{\alpha} u(x)=\int_{0}^{x} \frac{k(x, \xi)}{(x-\xi)^{\alpha}} u(\xi) d \xi, \quad 0<\alpha<1 .
$$

$y(x)$ is the solution to be determined and $g(x)$ and $k(x, \xi)$ are given functions. Throughout the present paper we assume that $g(x)$ and $k(x, \xi)$ are analytic on $0 \leq \xi \leq x$, and we fix some positive constant $X$ and solve the given equation over $0<x<X . \alpha$ is a parameter which determines the order of singularity at the right edge point $\xi=x$ and is assumed to be $0<\alpha<1$. Since the integral kernel has this sort of singularity the equation (1.1) is said to have a weakly singular kernel.

\footnotetext{
* Partially supported by the Grant-in-Aid for the 21st Century COE Research by the Ministry of Education, Culture, Sports, Science and Technology, and also by the Grand-in-Aid for Scientific Research (C) by Japan Society for the Promotion of Sciences.

${ }^{\dagger}$ In pin yin: Aheniyazi Nuermaimaiti.
} 
In general, the smoothness of the function $g(x)$ and that of $k(x, \xi)$ determine the smoothness of the solution on $x>0$. It is known that, under the assumption for the analyticity of $g$ and $k$, the solution $y(x)$ of (1.1) is weakly singular at the left edge point of the interval of integration $(0, x)$ [1], i.e. its derivative behaves

$$
y^{\prime}(x)=O\left(\frac{1}{x^{\alpha}}\right), \quad x \rightarrow 0_{+} .
$$

Since the singularity of the kernel in equation (1.1) will give some influence to the accuracy of the numerical solution, it will be helpful for us to have some knowledge about the singular behavior of the exact solution as mentioned above if we expect a high order convergence of the numerical method.

The purpose of the present paper is to present a method for numerical solution of Volterra integral equation of the type (1.1) using the Sinc collocation method based on the double exponential (DE) transformation. In 1992 B.V. Riley [5] proposed a Sinc collocation method for numerical solution of the equation (1.1) using the transformation $x=X e^{t} /\left(1+e^{t}\right)$. In the present paper we also give a method similar to that by Riley. However, there is a significant difference between our method and the method by Riley, i.e. we used the DE transformation proposed by H. Takahasi and M. Mori in 1974 [10] instead of $x=X e^{t} /\left(1+e^{t}\right)$. It will be shown that by using the DE transformation the efficiency of the numerical solution presents a remarkable improvement.

In the present paper we will follow the notation as in [5] for easy reference.

\section{DE transformation}

In this section we summarize the basic properties of the double exponential (DE) transformation. In the present paper we apply the DE transformation to two different components in the process of solving (1.1). The first component is expansion of the solution of equation (1.1) in terms of the Sinc functions, and the second component is numerical evaluation of the kernel integral (1.2).

First we introduce the DE transformation for the integral equation (1.1). Since the sinc function is defined over $-\infty<t<\infty$ we need some transformation from $-\infty<t<\infty$ onto $0<x<X$. For this purpose we employ the DE transformation

$$
x=\psi_{X}(t)=\frac{X}{2} \tanh \left(\frac{\pi}{2} \sinh t\right)+\frac{X}{2},
$$

or conversely

$$
t=\psi_{X}^{-1}(x)=\log \left(\frac{1}{\pi} \log \left(\frac{x}{X-x}\right)+\sqrt{\frac{1}{\pi^{2}}\left(\log \frac{x}{X-x}\right)^{2}+1}\right) .
$$


Then we have from $(2.1)$

$$
\psi_{X}(t)=X \frac{\exp \left(\frac{\pi}{2} \sinh t\right)}{\exp \left(\frac{\pi}{2} \sinh t\right)+\exp \left(-\frac{\pi}{2} \sinh t\right)}
$$

and

$$
\frac{d \psi_{X}(t)}{d t}=X \frac{\pi \cosh t}{\left(\exp \left(\frac{\pi}{2} \sinh t\right)+\exp \left(-\frac{\pi}{2} \sinh t\right)\right)^{2}} .
$$

Since the decay of derivative (2.4) is doubly exponential for large $|t|$ as

$$
\left|\frac{d \psi_{X}(t)}{d t}\right|=O\left(\exp \left(-\frac{\pi(1-\epsilon)}{2} \exp |t|\right)\right), \quad t \rightarrow \pm \infty,
$$

the transformation (2.1) is called the double exponential transformation [10]. $\epsilon$ is an arbitrarily small positive constant due to the increase by $\cosh t$ in the numerator of (2.4) for large $|t|$. Since $\epsilon$ is required only for mathematically rigorous discussion we set $\epsilon=0$ in actual computation.

Note that the array of points $t_{k}=k h, k=0, \pm 1, \pm 2, \ldots$ which lie equi-distantly on the entire real axis with an equal mesh size $h$ will be mapped by $\psi_{X}(t)$ onto an array of points $x_{k}=\psi_{X}(k h)$ which lie on the finite interval $0<x<X$. We call these points the Sinc points based on the DE transformation.

Next we consider application of the DE transformation to numerical evaluation of the kernel integral (1.2). In this case the interval of integration is $0<\xi<x$ and hence we have only to replace $X$ in $(2.3)$ with $x$ :

$$
\xi=\psi_{x}(\tau)=x \frac{\exp \left(\frac{\pi}{2} \sinh \tau\right)}{\exp \left(\frac{\pi}{2} \sinh \tau\right)+\exp \left(-\frac{\pi}{2} \sinh \tau\right)} .
$$

Let the integral which we want to evaluate be

$$
\int_{0}^{x} f(x, \xi) d \xi
$$

We assume here that $f(x, \cdot)$ is regular and bounded on $0<x$ and $f(\cdot, \xi)$ is integrable on $0<\xi<x$. In addition we assume that $f\left(x, \psi_{x}(\tau)\right) \psi_{x}{ }^{\prime}(\tau)$ is regular on the strip domain

$$
|\operatorname{Im} \tau|<d_{x} \quad\left(d_{x}>0\right)
$$

in the $\tau$ plane. Note that $d_{x}$ depends on $x$. If we apply variable transformation $\xi=\psi_{x}(\tau)$ to $(2.7)$ and integrate using the trapezoidal formula with an equal mesh size $\tilde{h}$ we have the following double exponential (DE) formula for (2.7) [10]:

$$
\begin{aligned}
& \int_{0}^{x} f(x, \xi) d \xi=\tilde{h} \sum_{k=-\infty}^{\infty} f\left(x, \psi_{x}(k \tilde{h})\right) \psi_{x}^{\prime}(k \tilde{h})+E_{\mathrm{de}}(\tilde{h}), \\
& E_{\mathrm{de}}(\tilde{h})=O\left(\exp \left(-\frac{2 \pi d_{x}}{\tilde{h}}\right)\right) .
\end{aligned}
$$


$E_{\mathrm{de}}(\tilde{h})$ in $(2.9)$ is the discretization error generated when $(2.7)$ is integrated using the DE formula.

If we write

$$
u_{k}=\frac{\exp \left(\frac{\pi}{2} \sinh k \tilde{h}\right)}{\exp \left(\frac{\pi}{2} \sinh k \tilde{h}\right)+\exp \left(-\frac{\pi}{2} \sinh k \tilde{h}\right)}
$$

then we see that

$$
x_{k}=\psi_{x}(k \tilde{h})=x u_{k}
$$

and

$$
\begin{aligned}
\psi_{x}^{\prime}(k \tilde{h})=\left(\frac{d \xi}{d \tau}\right)_{\tau=k \tilde{h}} & =\left.x \frac{\pi \cosh \tau}{\left(\exp \left(\frac{\pi}{2} \sinh \tau\right)+\exp \left(-\frac{\pi}{2} \sinh \tau\right)\right)^{2}}\right|_{\tau=k \tilde{h}} \\
& =x(\pi \cosh k \tilde{h}) u_{k}\left(1-u_{k}\right)
\end{aligned}
$$

hold at the Sinc points $\xi_{k}=\psi_{x}(k \tilde{h})=x u_{k}, k=0, \pm 1, \pm 2, \ldots$ Also we have from $(2.6)$

$$
x-\xi=x \frac{\exp \left(-\frac{\pi}{2} \sinh \tau\right)}{\exp \left(\frac{\pi}{2} \sinh \tau\right)+\exp \left(-\frac{\pi}{2} \sinh \tau\right)},
$$

and hence

$$
x-\xi_{k}=x\left(1-u_{k}\right)=x \frac{\exp \left(-\frac{\pi}{2} \sinh k \tilde{h}\right)}{\exp \left(\frac{\pi}{2} \sinh k \tilde{h}\right)+\exp \left(-\frac{\pi}{2} \sinh k \tilde{h}\right)} .
$$

\section{DE-Sinc approximation}

In this section we give an overview of the Sinc expansion and describe how we approximate the solution $y(x)$ of the equation (1.1) defined over a finite interval $0<x<X$ in the form of a Sinc expansion.

\subsection{Sinc cardinal series}

As is well known the sinc function is defined

$$
\operatorname{sinc}(t)=\frac{\sin \pi t}{\pi t}, \quad-\infty<t<\infty .
$$

Suppose that a function $v(t)$ is regular on the strip domain

$$
|\operatorname{Im} t|<d \quad(d>0)
$$

and satisfies

$$
\lim _{t \rightarrow \pm \infty} v(t)=0
$$


Then, it is known that under some additional mild analytical conditions $v(t)$ can be expressed in terms of the following sinc cardinal expansion using the sinc function (3.1):

$$
v(t)=\sum_{k=-\infty}^{\infty} v(k h) \operatorname{sinc}\left(\frac{t-k h}{h}\right)+E_{\text {sinc }}(h), \quad E_{\text {sinc }}(h)=O\left(\exp \left(-\frac{\pi d}{h}\right)\right) .
$$

See $[6,8,9]$ and references therein about the details of these conditions. The last term $E_{\text {sinc }}(h)$ is the discretization error due to the sinc expansion and we see that this error decreases very quickly as the mesh size $h$ becomes small. If we write the sum in the right hand side

$$
v_{h}(t)=\sum_{k=-\infty}^{\infty} v(k h) \operatorname{sinc}\left(\frac{t-k h}{h}\right),
$$

then $v_{h}(j h)=v(j h)$ holds, and hence $v_{h}(t)$ is an interpolation to $v(t)$ based on the points $t_{k}=k h, k=0, \pm 1, \ldots$ We also see that $v_{h}(t)$ converges to $v(t)$ as $h \rightarrow 0$.

\subsection{Sinc functions over a finite interval}

As seen from (3.1) the function $\operatorname{sinc}(t)$ is defined over the infinite interval $-\infty<t<\infty$. From this function we define the basis functions over the finite interval $0<x<X$ using the inverse function $\psi_{X}{ }^{-1}(x)$ of the DE transformation (2.3) in the following form:

$$
S(j, h, X)(x)=\operatorname{sinc}\left(\frac{\psi_{X}^{-1}(x)-j h}{h}\right), \quad j=0, \pm 1, \pm 2, \ldots
$$

The basis functions (3.6) are characterized by the parameter $h$, and we write them $S(j, h, X)(x)$ and capitalize as Sinc in order to discriminate them against the original function $\operatorname{sinc}(t)$. Since

$$
\begin{aligned}
S(j, h, X)\left(x_{k}\right) & =S(j, h, X)\left(\psi_{X}(k h)\right)=\operatorname{sinc}\left(\frac{\psi_{X}^{-1}\left(\psi_{X}(k h)\right)-j h}{h}\right) \\
& =\operatorname{sinc}(k-j)=\frac{\sin \pi(k-j)}{\pi(k-j)}
\end{aligned}
$$

holds, the basis functions (3.6) satisfy an orthogonal relation

$$
S(j, h, X)\left(x_{k}\right)=\delta_{k j}
$$

where $\delta_{k j}$ is the Kronecker's delta. If we apply the inverse transformation to (3.4) we have the following Sinc expansion over $0<x<X$ :

$$
V(x)=\sum_{k=-\infty}^{\infty} V\left(x_{k}\right) S(k, h, X)(x)+E_{\text {sinc }}(h),
$$

where

$$
V(x)=v\left(\psi_{X}^{-1}(x)\right), \quad x_{k}=\psi_{X}(k h)
$$




\subsection{Sinc expansion of the solution}

Now we are ready to approximate the solution $y(x)$ of equation (1.1) in the form of a Sinc expansion. Since $y(x)$ is defined on the finite interval $0<x<X$ we first need to transfer the finite interval $0<x<X$ onto the infinite interval $-\infty<t<\infty$ and, in addition, to make it satisfy (3.3) in order for the Sinc expansion (3.4) to be valid. For this purpose we define a function $Y(x)$ corresponding to the solution $y(x)$ of (1.1) in the following form:

$$
Y(x)=y(x)-\left(y(0)+\frac{y(X)-y(0)}{X} x\right) .
$$

We see $Y(x)$ satisfies

$$
Y(0)=Y(X)=0 .
$$

This condition corresponds to (3.3). Therefore $Y(x)$ can be expressed in terms of the basis functions $S(k, h, X), k=-\infty, \ldots, \infty$ in the following form of Sinc expansion in high accuracy if $h$ is small:

$$
Z_{\infty, \infty}(Y, h)(x) \equiv \sum_{j=-\infty}^{\infty} Y\left(\psi_{X}(j h)\right) S(j, h, X)(x) .
$$

We assume here more specifically that, for some positive constant $\alpha>0, Y(x)$ satisfies

$$
|Y(x)| \leq \begin{cases}C_{1} x^{1-\alpha}, & 0<x \leq \frac{1}{2} X \\ C_{1}^{\prime}(X-x), & \frac{1}{2} X<x \leq X .\end{cases}
$$

The behavior on $0<x \leq X / 2$ in (3.14) comes from the fact that $Y(x)$ approaches to 0 as $x^{1-\alpha}\left(x \rightarrow 0_{+}\right)$corresponding to (1.3), while the behavior on $X / 2<x \leq X$ comes from the fact that $Y(x)$ approaches to 0 at $x=X$ without any singularity as seen from (3.11). Then we can see that after we carry out DE transformation (2.3) $Y\left(\psi_{X}(t)\right)$ presents DE decay

$$
\left|Y\left(\psi_{X}(t)\right)\right|= \begin{cases}O\left(\exp \left(-\frac{\pi}{2}(1-\alpha) e^{|t|}\right)\right), & t \rightarrow-\infty \\ O\left(\exp \left(-\frac{\pi}{2} e^{|t|}\right)\right), & t \rightarrow+\infty\end{cases}
$$

When we use the expansion (3.13) for actual numerical computation we need to truncate the Sinc expansion appropriately at some lower limit $k=-M$ and at some upper limit $k=N$ :

$$
Z_{M, N}(Y, h)(x) \equiv \sum_{j=-M}^{N} Y\left(\psi_{X}(j h)\right) S(j, h, X)(x) .
$$


In the present situation, as seen from (3.15), the decay of the function $Y\left(\psi_{X}(t)\right)$ in the neighborhood of 0 , the left edge point, is slower than the decay in the neighborhood of $X$, the right edge point, and hence we choose $M$ and $N$ so that $M \geq N$ holds. Therefore, as in [5], we choose $M$, the larger number of terms of Sinc expansion, as a parameter in terms of which the performance of the present method is represented. Thus, since we truncate (3.16) at $k=-M$ we have a truncation error

$$
\begin{aligned}
E_{\text {trunc }}(M) & =Z_{\infty, \infty}(Y, h)(x)-Z_{M, N}(Y, h)(x) \\
& =O\left(\exp \left(-\frac{\pi}{2}(1-\alpha) \exp (M h)\right)\right)
\end{aligned}
$$

from (3.15). This truncation should be carried out in such a way that the truncation error $E_{\text {trunc }}(M)$ be approximately equal to the discretization error $E_{\text {sinc }}(h)$ in $(3.4)$, i.e.

$$
\exp \left(-\frac{\pi d}{h}\right)=\exp \left(-\frac{\pi}{2}(1-\alpha) \exp (M h)\right)
$$

which results in the following relation between the mesh size $h$ and the number of terms $M$ :

$$
h=\frac{1}{M} \log \frac{2 d M}{1-\alpha} .
$$

On the other hand if we truncate the sum (3.16) at $k=N$ we have a truncation error $E_{\text {trunc }}^{\prime}(N)=O\left(\exp \left(-\frac{\pi}{2} \exp N h\right)\right)$. This truncation should be done in such a way that this error be approximately equal to $E_{\text {trunc }}(M)$ in $(3.17)$, i.e. $(1-\alpha) \exp (M h)=$ $\exp (N h)$, which results in the following relation between $M$ and $N$ :

$$
N=\llbracket M+\frac{1}{h} \log (1-\alpha) \rrbracket+1 .
$$

【 』 denotes that fraction is discarded. Thus, if we first choose the lower limit $M$, next the mesh size $h$ to satisfy (3.19) and finally the upper limit $N$ to satisfy (3.20), then from (3.18) and (3.19) we obtain the following error bound for the approximation of $Y(x)$ in the form of (3.16) [7]:

$$
\left|Y(x)-Z_{M, N}(Y, h)(x)\right| \leq C_{2} \exp \left(-\frac{\pi d M}{\log (2 d M /(1-\alpha))}\right) .
$$

Note that from $(3.8) Z_{M, N}(Y, h)(x)$ satisfies

$$
Z_{M, N}(Y, h)\left(\psi_{X}(k h)\right)=Y\left(\psi_{X}(k h)\right), \quad k=-M, \ldots, N
$$

so that it is an interpolation to $Y(x)$.

$d$ is a parameter appearing in (3.2) and depends on the analyticity of $Y\left(\psi_{X}(t)\right)$. It is known that in case of the DE transformation (2.1) $d$ satisfies $d \leq \pi / 2$ [10]. However, it is usually difficult to get the exact value of $d$ in practical applications, and in the examples in Section 8 we use $d=\pi / 2$. 


\subsection{DE-Sinc collocation method}

Here we have come to the step where we consider an approximation to the solution $y(x)$ of the integral equation (1.1) in terms of the basis functions

$$
S(j, h, X), \quad j=-M, \ldots, N, \quad 1 \quad \text { and } \quad x / X
$$

in the following form:

$$
C_{M, N}(y, h)(x)=g(0)+\sum_{j=-M}^{N} Y\left(\psi_{X}(j h)\right) S(j, h, X)(x)+(y(X)-g(0)) \frac{x}{X} .
$$

That is, we replaced $Y(x)$ in (3.11) with the Sinc expansion (3.16). We also used $y(0)=g(0)$. This function interpolates $y(x)$ at $x=0, \psi_{X}(j h)(j=-M, \ldots, N)$ and $X$ as seen from $(3.8)$, i.e. $C_{M, N}(y, h)(x)$ satisfies

$$
C_{M, N}(y, h)(x)=y(x), \quad x=0, \psi_{X}(j h)(j=-M, \ldots, N), X .
$$

As we already mentioned we first choose the lower limit $M$, next compute the mesh size $h=\frac{1}{M} \log (2 d M /(1-\alpha))$ and finally take the upper limit $N=\llbracket M+$ $\frac{1}{h} \log (1-\alpha) \rrbracket+1$. Then from (3.11), (3.24) and (3.21) we see that the error of the approximation (3.24) satisfies

$$
\left|y(x)-C_{M, N}(y, h)(x)\right| \leq C_{3} \exp \left(-\frac{\pi d M}{\log (2 d M /(1-\alpha))}\right) .
$$

This indicates that as $M$ becomes large the error converges to 0 very quickly.

From this behavior of $C_{M, N}(y, h)$, we see that if we write

$$
y_{M, N}(x)=g(0)+\sum_{j=-M}^{N} c_{j} S(j, h, X)(x)+c_{N+1} \frac{x}{X}
$$

and determine the coefficients $c_{j}$ appropriately, $y_{M, N}(x)$ will approximate the solution $y(x)$ of (1.1) in high precision. The unknown value $y(X)$ at the right edge is included in the coefficients $c_{N+1}$ as $y(X)-g(0)$. The coefficients $c_{j}$ in the Sinc expansion $y_{M, N}(x)$ can be determined by the Sinc collocation method in such a way that $y_{M, N}(x)$ is forced to satisfy (1.1) at the Sinc points $x_{j}=\psi_{X}(j h)$ $(j=-M, \ldots, N), x_{N+1}=X$. We call the method DE-Sinc collocation method. The details of the DE-Sinc collocation method will be discussed in Section 5 . 


\section{Integration of the kernel by the DE formula}

In this section we consider numerical integration of the kernel (1.2). We regard integral (1.2) as a definite integral over the fixed interval $(0, x)$ and apply the DE formula with a mesh size $\tilde{h}$. Then from $(2.9)$ we have

$$
\begin{aligned}
& \int_{0}^{x} \frac{k(x, \xi)}{(x-\xi)^{\alpha}} y(\xi) d \xi \\
& =x \tilde{h} \sum_{j=-\infty}^{\infty} \frac{k\left(x, x u_{j}\right)}{x^{\alpha}\left(1-u_{j}\right)^{\alpha}} y\left(x u_{j}\right) \pi \cosh (j \tilde{h}) u_{j}\left(1-u_{j}\right)+E_{\mathrm{de}}(\tilde{h}) \\
& E_{\mathrm{de}}(\tilde{h})=O\left(\exp \left(-\frac{2 \pi d_{x}}{\tilde{h}}\right)\right) .
\end{aligned}
$$

$u_{k}$ is defined in (2.10). Note that the coefficients in the discretization error differ by a factor of two between $E_{\text {sinc }}(h)$ in $(3.4)$ and $E_{\mathrm{de}}(\tilde{h})$ in $(4.2)$.

We see that $k(x, \xi) y(\xi) /(x-\xi)^{\alpha}$ satisfies

$$
\left|\frac{k(x, \xi)}{(x-\xi)^{\alpha}} y(\xi)\right| \leq \begin{cases}C_{4}, & 0<\xi \leq \frac{1}{2} x \\ C_{4}^{\prime}(x-\xi)^{-\alpha}, & \frac{1}{2} x<\xi \leq x\end{cases}
$$

If we apply the DE transformation to (4.1) we see that

$$
\left|\frac{k\left(x, \psi_{x}(t)\right)}{\left(x-\psi_{x}(t)\right)^{\alpha}} y\left(\psi_{x}(t)\right) \psi_{x}^{\prime}(t)\right|= \begin{cases}O\left(\exp \left(-\frac{\pi(1-\epsilon)}{2} e^{|t|}\right)\right), & t \rightarrow-\infty \\ O\left(\exp \left(-\frac{\pi(1-\epsilon)}{2}(1-\alpha) e^{|t|}\right)\right), & t \rightarrow+\infty\end{cases}
$$

holds conversely to (3.15). In actual computation we need to truncate the infinite sum into a finite sum. Suppose that we truncate the infinite sum in the right hand side of (4.1) at some lower limit $j=-\tilde{N}$ and at some upper limit $j=\tilde{M}$ :

$$
x \tilde{h} \sum_{j=-\tilde{N}}^{\tilde{M}} \frac{k\left(x, x u_{j}\right)}{x^{\alpha}\left(1-u_{j}\right)^{\alpha}} y\left(x u_{j}\right) \pi \cosh (j \tilde{h}) u_{j}\left(1-u_{j}\right) .
$$

Since, as seen from (4.4), in the present case the decay of the integrand in the neighborhood of $x$, the right edge point, is slower, in contrast to (3.15), than the decay in the neighborhood of 0 , the left edge point, $\tilde{M} \geq \tilde{N}$ holds. And hence we should choose here $\tilde{M}$ as the parameter in terms of which the performance of the present DE formula is represented. Furthermore, in order to compute (3.27) and (4.5) with the same value of $M$ we take $M=\tilde{M}$, so that $M$ appears as the lower limit in (3.27) while as the upper limit in (4.5). 
If we equate the discretization error in (4.2) and the truncation error due to (4.5) under the assumption that $M$ is fixed as the same value in the expansion (3.27) we have the following relation between $\tilde{h}$ and $M$ :

$$
\tilde{h}=\frac{1}{M} \log \frac{4 d_{x} M}{1-\alpha} .
$$

Remember that, since $k\left(x, \psi_{x}(t)\right)$ includes $x, d_{x}$ in (4.6) depends on $x$. Therefore the truncation of the sum at the lower limit should be done in the same way as (3.20) at $j=-\tilde{N}$ where

$$
\tilde{N}=\llbracket M+\frac{1}{\tilde{h}} \log (1-\alpha) \rrbracket+1
$$

We define here

$$
L_{\tilde{N}, M}^{\alpha} y_{M, N}(x)=x^{1-\alpha} \tilde{h} \sum_{j=-\tilde{N}}^{M} k\left(x, x u_{j}\right) y_{M, N}\left(x u_{j}\right) \pi \cosh (j \tilde{h}) u_{j}\left(1-u_{j}\right)^{1-\alpha} .
$$

Then, if we write the error term (4.2) in the integral as a function of $M$ we have the following inequality for the error due to approximation by the DE formula:

$$
\left|L^{\alpha} y-L_{\tilde{N}, M}^{\alpha} y\right| \leq C_{5} \exp \left(-\frac{2 \pi d_{x} M}{\log \left(4 d_{x} M /(1-\alpha)\right)}\right) .
$$

If $d \leq 2 d_{x}(0<x<X)$ holds this error can be neglected compared with the error (3.26) of the Sinc expansion with the same value of $M$. And hence, we assume hereafter that

$$
d \leq 2 d_{x}, \quad 0<x<X
$$

holds and ignore the error (4.9).

Note that, as seen from (2.10), $u_{j}$ in (4.5) depends only on $\tilde{h}$ but not on $x$. Therefore, we do not have to evaluate the major part of the integrand repeatedly for different $x$ in the right hand side of $(4.5)$ if $k(x, \xi)$ does not depend on $\xi$ or $k(x, \xi)$ is a function of the form $\xi^{\beta}(x-\xi)^{\gamma}(\beta>-1, \gamma>\alpha-1)$. Also, in actual computation of (4.5), we should compute $1-u_{j}$ by using (2.14) in order to avoid loss of significant digits by cancellation in the neighborhood of $u_{j}=1$.

\section{Approximate solution by the DE-Sinc collocation method}

In this section we apply the DE-Sinc collocation method to the approximate solution of (1.1).

When we apply the collocation method we first choose $M$ in (3.27) as the upper limit of (4.8), next compute the mesh size as $\tilde{h}=\frac{1}{M} \log (4 d M /(1-\alpha))$, and finally take the lower limit $\tilde{N}=\llbracket M+\frac{1}{\tilde{h}} \log (1-\alpha) \rrbracket+1$. 
In order to determine the coefficients $c_{j}$ in the expansion (3.27) we replace $y(x)$ in (1.1) with $y_{M, N}(x)$ in (3.27) and $x$ with the Sinc point $x_{i}$. Then we have the following system of equations at each collocation point $x_{i}$ :

$$
y_{M, N}\left(x_{i}\right)=g\left(x_{i}\right)+L_{\tilde{N}, M}^{\alpha} y_{M, N}\left(x_{i}\right), \quad i=-M, \ldots, N+1 .
$$

If we replace $y_{M, N}\left(x_{i}\right)$ with the right hand side of (3.27) and define $f_{0}(x)=1$ and $f_{1}(x)=x / X$ we have

$$
\begin{gathered}
g(0)+\sum_{j=-M}^{N} c_{j} S(j, h, X)\left(x_{i}\right)+c_{N+1} \frac{x_{i}}{X}-g(0) L_{\tilde{N}, M}^{\alpha} f_{0}\left(x_{i}\right) \\
-\sum_{j=-M}^{N} c_{j} L_{\tilde{N}, M}^{\alpha} S(j, h, X)\left(x_{i}\right)-c_{N+1} L_{\tilde{N}, M}^{\alpha} f_{1}\left(x_{i}\right)=g\left(x_{i}\right),
\end{gathered}
$$

which can be reduced to

$$
\begin{aligned}
& c_{i}+\frac{x_{i}}{X} c_{N+1}-\sum_{j=-M}^{N} L_{\tilde{N}, M}^{\alpha} S(j, h, X)\left(x_{i}\right) c_{j}-L_{\tilde{N}, M}^{\alpha} f_{1}\left(x_{i}\right) c_{N+1} \\
& =g\left(x_{i}\right)+g(0)\left(L_{\tilde{N}, M}^{\alpha} f_{0}\left(x_{i}\right)-1\right)
\end{aligned}
$$

owing to (3.8). This equation can be written in the form of an $(M+N+2)$-nd order matrix equation

$$
\left(A_{M, N}-E_{M, N}\right) \boldsymbol{c}=\boldsymbol{g}
$$

where

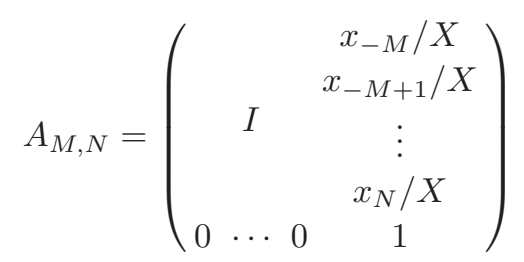

and $I$ is the unit matrix. The $(i, j)$ elements $e_{i j}$ of the matrix $E_{M, N}$ are given

$$
\begin{aligned}
& e_{i j}=L_{\tilde{N}, M}^{\alpha} S(j, h, X)\left(x_{i}\right), \quad i=-M, \ldots, N+1, \quad j=-M, \ldots, N, \\
& e_{i, N+1}=L_{\tilde{N}, M}^{\alpha} f_{1}\left(x_{i}\right), \quad i=-M, \ldots, N+1 .
\end{aligned}
$$

$\boldsymbol{c}$ and $\boldsymbol{g}$ are vectors

$$
\boldsymbol{c}=\left(c_{-M}, \ldots, c_{N+1}\right)^{T}
$$

and

$$
\boldsymbol{g}=\left(g_{-M}, \ldots, g_{N+1}\right)^{T},
$$


where the $i$-th element $g_{i}$ of $\boldsymbol{g}$ is

$$
g_{i}=g\left(x_{i}\right)+g(0)\left(L_{\tilde{N}, M}^{\alpha} f_{0}\left(x_{i}\right)-1\right), \quad i=-M, \ldots, N+1 .
$$

If we solve this system of linear equations for $c$ and substitute the solution for $c_{j}$ in (3.27) we have a numerical solution $y_{M, N}(x)$.

\section{Error bound}

In this section we derive inequalities which represent a typical error behavior of the approximate solution by the DE-Sinc method.

First we try to derive a bound of the error at each Sinc point $x_{i}$. From (1.1) and (5.1) we see that

$$
\begin{aligned}
C_{M, N}(y, h)\left(x_{i}\right)-y_{M, N}\left(x_{i}\right)= & y\left(x_{i}\right)-y_{M, N}\left(x_{i}\right)=L^{\alpha} y\left(x_{i}\right)-L_{\tilde{N}, M}^{\alpha}\left(x_{i}\right) y_{M, N}\left(x_{i}\right) \\
= & \left\{L^{\alpha} y\left(x_{i}\right)-L_{\tilde{N}, M}^{\alpha} C_{M, N}(y, h)\left(x_{i}\right)\right\} \\
& +L_{\tilde{N}, M}^{\alpha}\left\{C_{M, N}(y, h)\left(x_{i}\right)-y_{M, N}\left(x_{i}\right)\right\}
\end{aligned}
$$

because at the Sinc point $C_{M, N}(y, h)\left(x_{i}\right)=y\left(x_{i}\right)$ holds from (3.25). If we transpose the second term in the rightmost side to the leftmost side and write the result in a matrix form, we have from (3.24), (3.25) and (3.27)

$$
\left(A_{M, N}-E_{M, N}\right)(\boldsymbol{b}-\boldsymbol{c})=\boldsymbol{L}^{\alpha} y-\boldsymbol{L}_{\tilde{N}, M}^{\alpha} C_{M, N}(y, h),
$$

and hence

$$
\left(A_{M, N}-E_{M, N}\right)(\boldsymbol{b}-\boldsymbol{c})=\left\{\boldsymbol{L}^{\alpha} y-\boldsymbol{L}_{\tilde{N}, M}^{\alpha} y\right\}+\boldsymbol{L}_{\tilde{N}, M}^{\alpha}\left\{y-C_{M, N}(y, h)\right\},
$$

where $\boldsymbol{c}$ is defined in (5.8) and

$$
\boldsymbol{b}=\left(Y\left(x_{-M}\right), \ldots, Y\left(x_{N}\right), Y(X)-g(0)\right)^{T} .
$$

Note that $(\boldsymbol{b}-\boldsymbol{c})_{i}=Y\left(x_{i}\right)-c_{i}$ is the error at the Sinc point $x_{i} . \boldsymbol{L}^{\alpha} y$ and $\boldsymbol{L}_{\tilde{N}, M}^{\alpha}(y, h)$ are vectors whose $i$-th elements are $L^{\alpha} y\left(x_{i}\right)$ and $L_{\tilde{N}, M}^{\alpha} C_{M, N}(y, h)\left(x_{i}\right)$, respectively. We write here

$$
\mu_{M}=\left\|\left(A_{M, N}-E_{M, N}\right)^{-1}\right\| .
$$

In the present paper $\|\cdot\|$ denotes the infinity vector norm and the associated matrix norm. From (6.3) we see that

$$
\|\boldsymbol{b}-\boldsymbol{c}\| \leq \mu_{M}\left\{\left\|\boldsymbol{L}^{\alpha} y-\boldsymbol{L}_{\tilde{N}, M}^{\alpha} y\right\|+\left\|\boldsymbol{L}_{\tilde{N}, M}^{\alpha}\left\{y-C_{M, N}(y, h)\right\}\right\|\right\}
$$

holds. And hence from (4.9), which can be ignored under the assumption (4.10), and from (3.26) we finally have a bound for the maximum error at the Sinc points:

$$
\max _{-M \leq i \leq N+1}\left|y\left(x_{i}\right)-c_{i}\right| \leq C_{6} \mu_{M} \exp \left(-\frac{\pi d M}{\log (2 d M /(1-\alpha))}\right) .
$$

This represents a nearly exponential convergence. 
If an estimate of $\mu_{M}$ is available we immediately have an error bound from (6.7). It is not impossible to get a bound of $\mu_{M}=\left\|\left(A_{M, N}-E_{M, N}\right)^{-1}\right\|$ in a mathematically rigorous manner, but it is usually difficult or unrealistic as is discussed in [5]. Instead, in the present paper we monitor the value of $\mu_{M}$ when we actually solve (5.4) and judge whether the numerical solution obtained is reliable or not. If $\mu_{M}$ is not too large we can say that the solution obtained is reliable.

In order to solve the system of linear equations (5.4) we employed the LU decomposition code DECOMP from the book [2]. DECOMP computes $\| A_{M, N}-$ $E_{M, N} \|$ which we denotes ANORM as well as an estimate of $\left\|\left(A_{M, N}-E_{M, N}\right)^{-1}\right\|$ which we denote AINORM, and returns COND $=\mathrm{ANORM} * \mathrm{AINORM}$ as an estimate of the condition number of the matrix $A_{M, N}-E_{M, N}$. Although AINORM usually gives a good estimate of $\mu_{M}$ DECOMP does not return it, and hence we modified DECOMP so that it also returns AINORM. In Table 1 in Section 8 we show the value of COND and AINORM when we solved the equation for Example 1. We note here that there is a small difference between the definition of the matrix norm in the present method and that used in DECOMP. See [4] for detailed discussion about the difference.

In order to get a bound of the error at an arbitrary point $x$ we use

$$
\left|y(x)-y_{M, N}(x)\right| \leq\left|y(x)-C_{M, N}(y, h)(x)\right|+\left|C_{M, N}(y, h)(x)-y_{M, N}(x)\right| .
$$

Here we note that it can be shown [5] that the Sinc functions $S(j, h, X)$ satisfy

$$
\sum_{j=-M}^{N}|S(j, h, X)(x)| \leq C_{7} \log M
$$

for large $M$, and hence

$$
\begin{aligned}
& \left|C_{M, N}(y, h)(x)-y_{M, N}(x)\right| \\
& =\left|\sum_{j=-M}^{N}\left(y\left(x_{j}\right) S(j, h, X)(x)-c_{j} S(j, h, X)(x)\right)+\left(y(X)-g(0)-c_{N+1}\right) \frac{x}{X}\right| \\
& \leq \sum_{j=-M}^{N}\left|y\left(x_{i}\right)-c_{j}\right||S(j, h, X)(x)|+\left|y(X)-g(0)-c_{N+1}\right| \\
& \leq C_{8} \log M\|\boldsymbol{b}-\boldsymbol{c}\|
\end{aligned}
$$

holds. Then from (3.26), (6.7) and (6.8) we finally obtain

$$
\left|y(x)-y_{M, N}(x)\right| \leq C_{9} \mu_{M} \log M \exp \left(-\frac{\pi d M}{\log (2 d M /(1-\alpha))}\right)
$$

for all $0 \leq x \leq X$ under the assumption (4.10). 


\section{SE transformation}

In 1992 B.V. Riley [5] proposed a method for solving Volterra integral equations with weakly singular kernel based on the transformation

$$
x=\psi_{X}^{(\mathrm{S})}(t)=X \frac{\exp (t)}{1+\exp (t)} .
$$

In this case the derivative $d \psi_{X}^{(\mathrm{S})}(t) / d t$ behaves

$$
\frac{d \psi_{X}^{(\mathrm{S})}(t)}{d t}=X \frac{\exp (t)}{(1+\exp (t))^{2}}=O(\exp (-|t|)), \quad t \rightarrow \pm \infty
$$

Since this decay is singly exponential we call (7.1) the single exponential (SE) transformation.

Let $S^{(\mathrm{S})}(j, h, X)$ be the Sinc function based on the SE transformation and write

$$
y_{M, N}^{(\mathrm{S})}(x)=g(0)+\sum_{j=-M}^{N} c_{j}^{(\mathrm{S})} S^{(\mathrm{S})}(j, h, X)(x)+c_{N+1}^{(\mathrm{S})} \frac{x}{X} .
$$

In case of the SE transformation we first choose $M$ and compute $h, N$ and $\tilde{h}$ as follows [5]:

$$
h=\sqrt{\frac{\pi d}{(1-\alpha) M}}, \quad N=\llbracket(1-\alpha) M \rrbracket+1, \quad \tilde{h}=\sqrt{\frac{2 \pi d}{(1-\alpha) M}} .
$$

Then, if we replace DE transformation with SE transformation and apply the Sinc collocation method developed in the preceding sections with the parameters selected above, we obtain a numerical solution $y_{M, N}^{(\mathrm{S})}(x)$ in (7.3). In [5] it is also proved that the maximum error of the numerical solution $y_{M, N}^{(\mathrm{S})}(x)$ at the Sinc points $x_{i}^{(\mathrm{S})}=\psi_{X}^{(\mathrm{S})}(i h)$ satisfies

$$
\max _{-M \leq i \leq N+1}\left|y\left(x_{i}^{(\mathrm{S})}\right)-c_{i}^{(\mathrm{S})}\right| \leq C_{10} \sqrt{M} \exp (-\sqrt{\pi d(1-\alpha) M})
$$

and that the error at an arbitrary point $x$ satisfies

$$
\left|y(x)-y_{M, N}^{(\mathrm{S})}(x)\right| \leq C_{11} \sqrt{M} \log M \exp (-\sqrt{\pi d(1-\alpha) M})
$$

for all $0 \leq x \leq X$.

Riley [5] derived an estimate $\mu_{M} \leq 4$ under certain assumptions, so that (7.5) and (7.6) do not include $\mu_{M}$. But these assumptions have some ambiguous expression such as "for all $M$ in a practical range." And hence we did not adopted the method of proof in [5] and instead we monitored $\mu_{M}$ during numerical computation of solution. 


\section{Numerical examples}

In this section we apply the method developed in the previous sections to two examples.

In order to obtain numerical solutions we need to solve $(M+N+2)$-nd order system of linear equations for $c$ as is already mentioned. We employed the LU decomposition code DECOMP for that purpose and solved the problems with quadruple precision arithmetic because we want to emphasize the high precision of the numerical solution obtained by the present method. We used Fujitsu Fortran compiler whose machine epsilon in quadruple precision arithmetic is about $9.63 \times 10^{-35}$. In every example we used $d=\pi / 2$.

Since in both examples the exact solution $y(x)$ is known, we computed the error

$$
\max _{-M \leq i \leq N+1}\left|y\left(x_{i}\right)-y_{M, N}\left(x_{i}\right)\right|
$$

of the approximate solution $y_{M, N}\left(x_{i}\right)$ based on the DE transformation at the Sinc points $x_{i}(i=-M,-M+1, \ldots, N+1)$. Also we computed the error of the solution based on the SE transformation

$$
\max _{-M \leq i \leq N+1}\left|y\left(x_{i}^{(\mathrm{S})}\right)-y_{M, N}^{(\mathrm{S})}\left(x_{i}^{(\mathrm{S})}\right)\right|
$$

at the Sinc points $x_{i}^{(\mathrm{S})}(i=-M,-M+1, \ldots, N+1)$.

Example 1.

$$
\begin{aligned}
& y(x)=\sqrt{x}+\frac{\pi x}{2}-\int_{0}^{x} \frac{y(t)}{\sqrt{x-t}} d t, \quad 0 \leq x \leq X, \\
& \text { exact solution: } \quad y(x)=\sqrt{x} .
\end{aligned}
$$

This problem is from [5]. In Fig. 1 we show the error (8.1) with $X=1$ and with $X=8$ for $M=2,4,8,16,32$ and 64 . The curve marked as $\operatorname{DE}(X=1)$ is for the error by the present method based on the DE transformation with $X=1$, while the curve marked as $\mathrm{DE}(X=8)$ is for the error with $X=8$. This shows that our method gives a good solution even for $X$ as large as 8 . In this figure we also find a curve marked as SE $(X=1)$ which shows the error by the method based on the SE transformation with $X=1$. In this figure the convergence behavior (6.7) of the error to zero with the DE transformation and also (7.5) with the SE transformation are clearly observed. It is apparent that the convergence rate by the DE transformation is much faster than that by the SE transformation.

In Table 1 we show COND, an estimate of the condition number, and AINORM, an estimate $\mu_{M}$, of the matrix $A_{M, N}-E_{M, N}$ returned by DECOMP in case of the $\mathrm{DE}$ transformation with $X=1$. In this table we see that $\mu_{M}=$ $\left\|\left(A_{M, N}-E_{M, N}\right)^{-1}\right\|$ is kept reasonably small even for $M$ as large as 64 . 


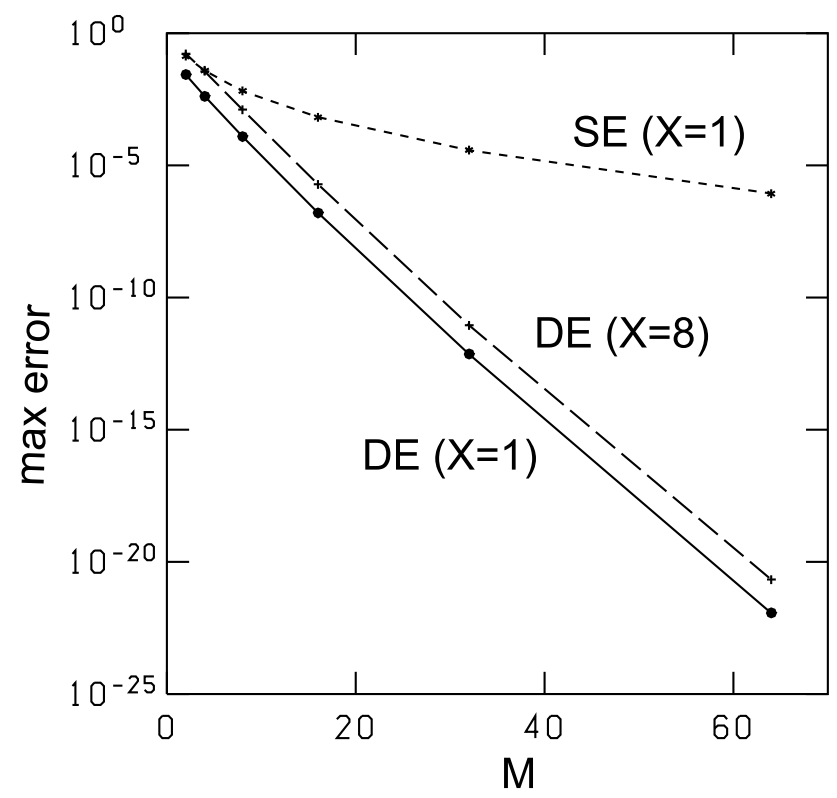

Fig. 1. Maximum error in Example 1.

Table 1. COND and AINORM for the matrix $A_{M, N}-E_{M, N}$ by the DE-Sinc method in Example 1.

\begin{tabular}{|c|c|c|}
\hline$M$ & COND (DE) & AINORM (DE) \\
\hline 2 & $1.55 \mathrm{e}+01$ & $3.37 \mathrm{e}+00$ \\
\hline 4 & $2.73 \mathrm{e}+01$ & $4.01 \mathrm{e}+00$ \\
\hline 8 & $6.08 \mathrm{e}+01$ & $6.00 \mathrm{e}+00$ \\
\hline 16 & $1.54 \mathrm{e}+02$ & $8.78 \mathrm{e}+00$ \\
\hline 32 & $5.39 \mathrm{e}+02$ & $1.68 \mathrm{e}+01$ \\
\hline 64 & $1.74 \mathrm{e}+03$ & $3.16 \mathrm{e}+01$ \\
\hline
\end{tabular}

In case of $X=1$ we divided the interval $0 \leq x \leq X=1$ into 1000 equal subintervals with mesh size 0.001 and computed also the error $\left|y\left(x_{i}\right)-y_{M, N}\left(x_{i}\right)\right|$ on the equal mesh $x_{i}=i / 1000,1 \leq i \leq 999$ for each $M$. In Table 2 we show the maximum error on the Sinc mesh $X_{j}=\psi(j h), j=-M, \ldots, N$ and that on the equal mesh together with the points at which the maximum is attained.

In Fig. 2 we show the graph of the exact solution $y(x)=\sqrt{x}$ as well as those of the numerical solutions with $M=2$ and $M=4$. The solid curve corresponds to $y(x)$, the dotted curve to $M=2$ and the broken curve to $M=4$. We see that even with $M=4$ we obtain a fairly good numerical solution. As $M$ becomes larger the numerical solution approximates $y(x)$ better, and with $M=8$ we are almost unable to discriminate the numerical solution from the exact solution on the graph. 
Table 2. The point and the error at which the maximum is attained on DE-Sinc mesh and on equal mesh in Example 1.

\begin{tabular}{|c|c|c|c|c|}
\hline & \multicolumn{2}{|c|}{ on DE-Sinc mesh } & \multicolumn{2}{c|}{ on equal mesh } \\
\hline$M$ & at $x$ & error & at $x$ & error \\
\hline 2 & 0.994 & $2.70 \mathrm{e}-02$ & 0.828 & $7.03 \mathrm{e}-02$ \\
\hline 4 & 0.500 & $4.05 \mathrm{e}-03$ & 0.741 & $1.39 \mathrm{e}-02$ \\
\hline 8 & 0.832 & $1.23 \mathrm{e}-04$ & 0.912 & $4.27 \mathrm{e}-04$ \\
\hline 16 & 0.715 & $1.59 \mathrm{e}-07$ & 0.790 & $6.62 \mathrm{e}-07$ \\
\hline 32 & 0.628 & $7.15 \mathrm{e}-13$ & 0.681 & $3.64 \mathrm{e}-12$ \\
\hline 64 & 0.573 & $1.15 \mathrm{e}-22$ & 0.452 & $6.99 \mathrm{e}-18$ \\
\hline
\end{tabular}

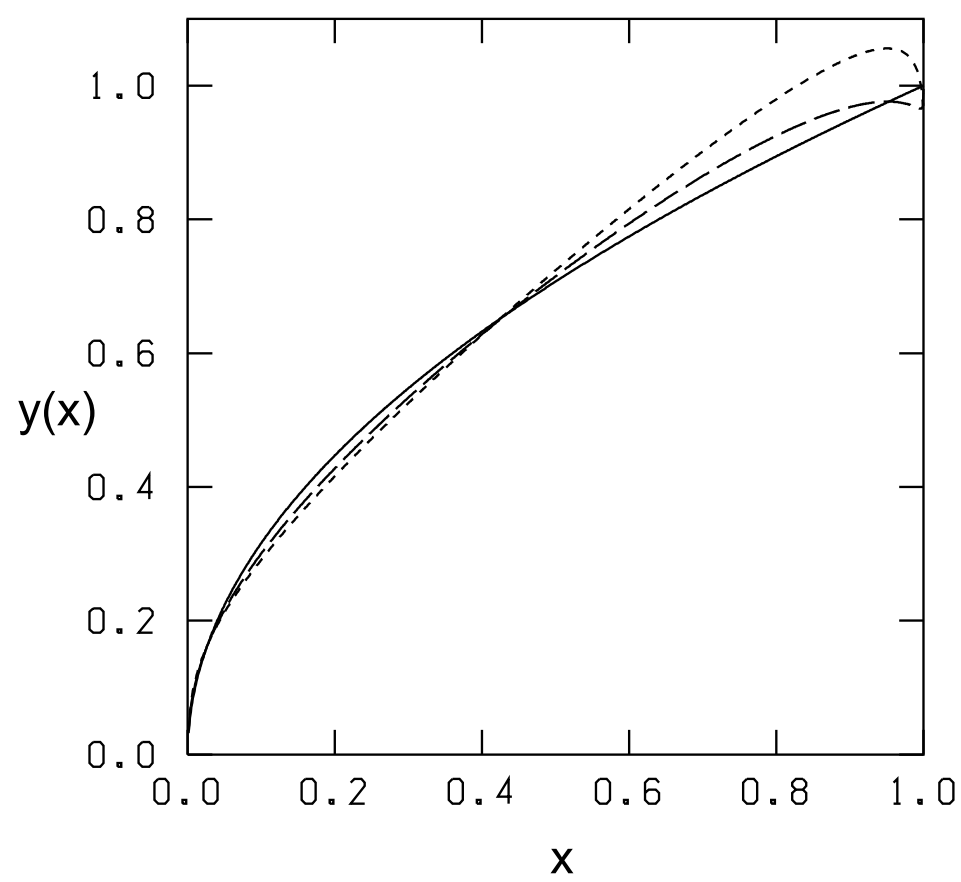

Fig. 2. Exact solution $y=\sqrt{x}$ and the numerical solutions for $M=2$ and $M=4$ in Example 1.

Example 2.

$y(x)=x^{2}+\int_{0}^{x}(10 \exp (-(x-t))-6 \exp (-2(x-t))) y(t) d t, \quad 0 \leq x \leq X$,

exact solution: $\quad y(x)=\frac{15 \exp (4 x)}{112}+\frac{4 \exp (-3 x)}{189}-\frac{x^{2}}{6}-\frac{17 x}{36}-\frac{67}{432}$. 
In 2003 Muhammad and Mori [3] proposed a method for indefinite numerical integration based on the DE transformation, and in $2005 \mathrm{M}$. Muhammad et al. [4] applied this method to numerical solution of Volterra integral equations of the second kind with a regular kernel. On the other hand, even if $\alpha=0$ holds in (1.1), i.e. the equation in question is a Volterra integral equation with a regular kernel, the present method can of course be applied to numerical solution of the equation. Example 2 is a problem which meets the situation. We set $\alpha=0$ and chose the parameters as follows:

$$
h=\frac{1}{M} \log (2 d M), \quad \tilde{h}=\frac{1}{M} \log (4 d M), \quad N=\tilde{N}=M+1 .
$$

We solved the problem with $X=1$ using the present method and the one proposed by Muhammad et al. [4] for $M=2,4,8,16,32,64,96$ and 128. In Fig. 3 we show the error of numerical solution by these two methods as well as the error by the method based on the SE transformation which we marked as SE-Sinc. DE-Sinc indicates the error by the present method, while DE-indef indicates the error by the method in [4]. We see that the methods based on the DE transformation give a good result, although the present method presents a slightly better convergence. In Table 3 we show COND and AINORM obtained by the DE-Sinc method in Example 2. From this table we see that $\mu_{M}$ is kept small also in this problem.

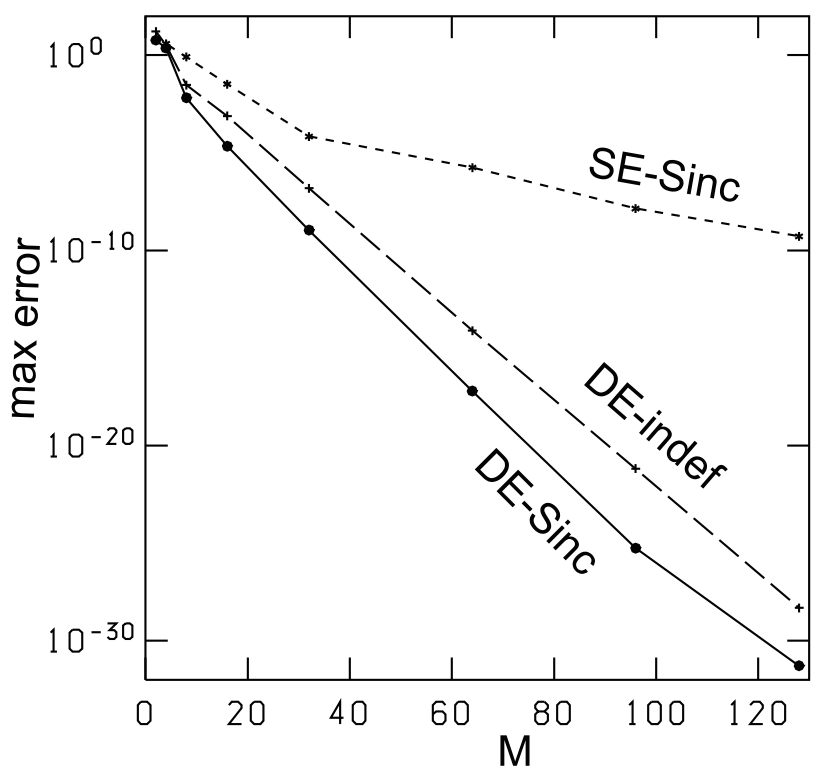

Fig. 3. Maximum error in Example 2. 
Table 3. COND and AINORM for the matrix $A_{M, N}-E_{M, N}$ by the DE-Sinc method in Example 2.

\begin{tabular}{|c|c|c|}
\hline$M$ & COND (DE) & AINORM (DE) \\
\hline 2 & $1.39 \mathrm{e}+02$ & $1.86 \mathrm{e}+01$ \\
\hline 4 & $3.18 \mathrm{e}+02$ & $4.40 \mathrm{e}+01$ \\
\hline 8 & $3.90 \mathrm{e}+02$ & $3.79 \mathrm{e}+01$ \\
\hline 16 & $3.92 \mathrm{e}+02$ & $2.24 \mathrm{e}+01$ \\
\hline 32 & $3.90 \mathrm{e}+02$ & $1.20 \mathrm{e}+01$ \\
\hline 64 & $4.39 \mathrm{e}+02$ & $7.76 \mathrm{e}+00$ \\
\hline 128 & $4.94 \mathrm{e}+02$ & $4.91 \mathrm{e}+00$ \\
\hline
\end{tabular}

Acknowledgment. The authors would like to express their thanks to the referee for giving precious comments.

\section{References}

[ 1 ] H. Brunner and P.J. van der Houwen, The Numerical Solution of Volterra Equations. NorthHolland, Amsterdam, 1986.

[2] G.E. Forsythe, M.A. Malcolm and C.B. Moler, Computer Methods for Mathematical Computations. Prentice-Hall, Inc., Englewood Cliffs, New Jersey, 1977.

[ 3 ] M. Muhammad and M. Mori, Double exponential formulas for numerical indefinite integration. J. Comput. Appl. Math., 161 (2003), 431-448.

[ 4 ] M. Muhammad, A. Nurmuhammad, M. Mori and M. Sugihara, Numerical solution of integral equations by means of the Sinc collocation method based on the double exponential transformation. J. Comput. Appl. Math., 177 (2005), 269-286.

[ 5 ] B.V. Riley, The numerical solution of Volterra integral equations with nonsmooth solutions based on sinc approximation. J. Comput. Appl. Math., 9 (1992), 249-257.

[6] F. Stenger, Numerical Methods Based on Sinc and Analytic Functions. Springer-Verlag, New York, 1993.

[ 7 ] M. Sugihara, Near optimality of the Sinc approximation. Math. Comput., 72 (2003), $767-786$.

[ 8 ] H. Takahasi, Complex function theory and numerical analysis (in Japanese). Kokyuroku, RIMS Kyoto Univ., 253 (1975), 24-37.

[ 9 ] H. Takahasi, Complex function theory and numerical analysis. Publ. RIMS Kyoto Univ., 41 (2005), 979-988.

[10] H. Takahasi and M. Mori, Double exponential formulas for numerical integration. Publ. RIMS Kyoto Univ., 9 (1974), 721-741. 
